PROCEEDINGS OF THE

AMERICAN MATHEMATICAL SOCIETY

Volume 129, Number 12, Pages 3665-3669

S 0002-9939(01)06020-8

Article electronically published on April 26, 2001

\title{
SOME GENERALIZATIONS OF CHIRKA'S EXTENSION THEOREM
}

\author{
GAUTAM BHARALI
}

(Communicated by Steven R. Bell)

\begin{abstract}
In this paper, we generalize Chirka's theorem on the extension of functions holomorphic in a neighbourhood of $S \cup(\partial D \times D)$ - where $D$ is the open unit disc in $\mathbb{C}$ and $S$ is the graph of a continuous $D$-valued function on $\bar{D}$ - to higher dimensions, for certain classes of graphs $S \subseteq \bar{D} \times D^{n}, n>1$. In particular, we show that Chirka's extension theorem generalizes to configurations in $\mathbb{C}^{n+1}, n>1$, involving graphs of (non-holomorphic) polynomial maps with small coefficients.
\end{abstract}

\section{THE MAIN THEOREM}

This paper is motivated by an article by Chirka [1] (also see [2]) in which he proves the following result (in what follows, $D$ will denote the open unit disc in $\mathbb{C}$, while $D_{r}$ will denote the open disc of radius $r$, centered at $0 \in \mathbb{C}$ ) :

Theorem 1.1 (Chirka). Let $\phi: \bar{D} \rightarrow \mathbb{C}$ be a continuous function having $\sup _{z \in \bar{D}}|\phi(z)|<1$ and let $S$ be its graph. Let $\Omega$ be a connected neighbourhood of $S \cup(\partial D \times D)$ contained in $\left\{(z, w) \in \mathbb{C}^{2}:|w|<1\right\}$. If $f \in \mathcal{O}(\Omega)$, then $f$ extends holomorphically to $D^{2}$.

In the same paper, Chirka asks if the above theorem is valid in the multidimensional case [1. question 1], i.e. when $S$ is the graph of a continuous $D^{n}$-valued map with $n>1$. Rosay [3] showed that the theorem fails in general for higher dimensions. A natural question that arises is whether holomorphic extension to $D^{n+1}, n>1$, occurs when the component functions of the $D^{n}$-valued map defining our graph are small in some appropriate sense (for instance, when the graph is a sufficiently small perturbation of a holomorphic graph). We are able to answer Chirka's question in the affirmative for the class of graphs described in Theorem 1.3 below. Before stating that theorem, however, we state the following proposition, which is a special case of Theorem 1.3. We highlight this as a separate proposition because of the clarity of its statement.

Received by the editors May 1, 2000.

2000 Mathematics Subject Classification. Primary 32D15.

Key words and phrases. Holomorphic extension. 
Proposition 1.2. Let $S$ be the graph of a map $\left(P_{1}, \ldots, P_{n}\right): \bar{D} \rightarrow \mathbb{C}^{n}$, where $P_{k}, k=1, \ldots, n$, are polynomials

$$
P_{k}(z)=\sum_{|\alpha|=0}^{N} C_{\alpha}^{(k)} z^{\alpha_{1}} \bar{z}^{\alpha_{2}}
$$

with the property $\sum_{|\alpha| \leq N}\left|C_{\alpha}^{(k)}\right|<1, k=1, \ldots, n$. Let $\Omega$ be a connected neighbourhood of $S \cup\left(\partial D \times D^{n}\right)$ contained in $\left\{(z, w) \in \mathbb{C} \times \mathbb{C}^{n} \mid w \in D^{n}\right\}$. If $f \in \mathcal{O}(\Omega)$, then $f$ extends holomorphically to $D^{n+1}$.

The main result is as follows:

Theorem 1.3. Let $S$ be the graph of the map $\left(\phi_{1}, \ldots, \phi_{n}\right): \bar{D} \rightarrow \mathbb{C}^{n}$, where for each $\phi_{k}, k=1, \ldots, n$ :

$$
\phi_{k} \in\left[\left\{(z, \bar{z}) \mapsto \psi(z, \bar{z}) \mid \psi \in \mathcal{O}\left(D^{2}\right) \text { and } \sup _{(z, \xi) \in D^{2}}|\psi(z, \xi)|<1\right\} \cap \mathcal{C}(\bar{D} ; D)\right] .
$$

If $\Omega$ is a connected neighbourhood of $S \cup\left(\partial D \times D^{n}\right)$ contained in $\{(z, w) \in \mathbb{C} \times$ $\left.\mathbb{C}^{n} \mid w \in D^{n}\right\}$ and if $f \in \mathcal{O}(\Omega)$, then $f$ extends holomorphically to $D^{n+1}$.

Remark. The proof of the above statement (and all its corollaries) can actually be worked out without appealing to the Hartogs-Chirka theorem (i.e. Theorem 1.1). As we shall see in the proof below, our problem reduces to the case of $n=1$ and the graph of $\phi(z)=\bar{z}$. At this stage, we could simply invoke Theorem 1.1 to complete the proof. However, we provide an elementary proof of analytic continuation to $D^{2}$ for the aforementioned case (see Lemma 1.4 below).

Proof of Theorem 1.3. We first observe that without loss of generality, it suffices to let $\phi_{k}$ be polynomials $\phi_{k}(z, \bar{z})=P_{k}(z, \bar{z})$, with $\sup _{(z, \xi) \in \bar{D}^{2}}\left|P_{k}(z, \xi)\right|<1$. This is because the functions $\phi_{k}$ can be approximated arbitrarily closely by such polynomials.

Let $\delta>0$ be small enough that

$$
\begin{gathered}
\left\{\left(z, w_{1}, \ldots, w_{n}\right) \in \mathbb{C}^{n+1}:|z|<1,\left|w_{k}-P_{k}(z, \bar{z})\right|<\delta, k=1, \ldots, n\right\} \subseteq \Omega, \\
R_{\delta}=\left\{(z, w) \in \mathbb{C}^{n+1}: 1-\delta<|z|<1+\delta, w \in D^{n}\right\} \subseteq \Omega .
\end{gathered}
$$

There is an $\epsilon_{0}, 0<\epsilon_{0}<\delta / 2$, sufficiently small that for $\left|w_{k}\right|<\epsilon_{0}, k=1, \ldots, n$, $|z|<1$ and $|\xi-\bar{z}|<\epsilon_{0},\left|\left\{w_{k}+P_{k}(z, \xi)\right\}-P_{k}(z, \bar{z})\right|<\delta, k=1, \ldots, n$. So, for each fixed $w \in D_{\epsilon_{0}}^{n}$, for $|z|<1,|\xi-\bar{z}|<\epsilon_{0}$, we can define

$$
H(z, \xi, w)=f\left(z, w_{1}+P_{1}(z, \xi), \ldots, w_{n}+P_{n}(z, \xi)\right) .
$$

Notice that the hypothesis $\sup _{(z, \xi) \in \bar{D}^{2}}\left|P_{k}(z, \xi)\right|<1$ allows us to define, for each $w \in D_{\epsilon_{0}}^{n}$, the function

$$
(z, \xi) \mapsto H(z, \xi, w),
$$

which is well defined and holomorphic in $\left\{|z|<1,|\xi-\bar{z}|<\epsilon_{0}\right\} \cup\{1-\delta<|z|<$ $1+\delta,|\xi| \leq 1\} \subseteq \mathbb{C}^{2}$

At this stage, we need the following lemma, which is a special case of Theorem 1.1, but for which we provide a direct and elementary proof. We defer its proof until the end of this demonstration. 
Lemma 1.4. Let $S$ be the graph of the function $\phi(z)=\bar{z}$ over $\bar{D}$. Let $\omega$ be a neighbourhood of $S \cup(\partial D \times \bar{D})$, and $V$ the connected component of $\omega \cap D^{2}$ containing $\partial D \times D$. If $f \in \mathcal{O}(\omega)$, then $\left.f\right|_{V}$ extends holomorphically to a neighbourhood of $D^{2}$.

By Lemma 1.4, we can define a function $\tilde{H}$, such that for each $w \in D_{\epsilon_{0}}^{n}$

$$
(z, \xi) \mapsto \tilde{H}(z, \xi, w)
$$

is a holomorphic extension of the function given by (ii) to $D^{2}$. As $\{H(\cdot, \cdot, w)\}_{\left|w_{k}\right|<\epsilon_{0}}$ is an analytic family, so is $\{\tilde{H}(\cdot, \cdot, w)\}_{\left|w_{k}\right|<\epsilon_{0}}$, whereby we have the fact that

$$
(z, \xi, w) \mapsto \tilde{H}\left(z, \xi, w_{1}-P_{1}(z, \xi), \ldots, w_{n}-P_{n}(z, \xi)\right)
$$

is holomorphic in $\left\{|z|<1,|\xi|<1,\left|w_{k}-P_{k}(z, \xi)\right|<\epsilon_{0}\right\}$.

Now define

$$
\tilde{f}(z, w)=\tilde{H}\left(z, 0, w_{1}-P_{1}(z, 0), \ldots, w_{n}-P_{n}(z, 0)\right)
$$

where $\tilde{f}$ is defined and holomorphic in $\left\{(z, w):|z|<1,\left|w_{k}-P_{k}(z, 0)\right|<\epsilon_{0}\right\}$. But $\tilde{f}$ is also defined in $R_{\delta}$, and since for $1-\delta<|z|<1+\delta$,

$\tilde{H}\left(z, 0, w_{1}-P_{1}(z, 0), \ldots, w_{n}-P_{n}(z, 0)\right)=H\left(z, 0, w_{1}-P_{1}(z, 0), \ldots, w_{n}-P_{n}(z, 0)\right)$,

we have

$$
\begin{aligned}
\tilde{f}\left(z, w_{1}, \ldots, w_{n}\right) & =\tilde{H}\left(z, 0, w_{1}-P_{1}(z, 0), \ldots, w_{n}-P_{n}(z, 0)\right) \\
& =H\left(z, 0, w_{1}-P_{1}(z, 0), \ldots, w_{n}-P_{n}(z, 0)\right) \\
& =f\left(z, w_{1}, \ldots, w_{n}\right) \quad[\text { by (i) }]
\end{aligned}
$$

for all $(z, w) \in R_{\delta}$. So, $\left.\tilde{f}\right|_{R_{\delta}}=f_{R_{\delta}}$. We therefore conclude that

$$
\tilde{f} \text { is holomorphic in a neighbourhood of }\left(\partial D \times D^{n}\right) \cup \sigma
$$

where $\sigma$ is the graph of the holomorphic map $z \mapsto\left(P_{1}(z, 0), \ldots, P_{n}(z, 0)\right)$ over $\bar{D}$. By the classical theorem of Hartogs, $\tilde{f}$ extends to $F \in \mathcal{O}\left(D^{n+1}\right)$, and $\left.F\right|_{R_{\delta}}=\left.f\right|_{R_{\delta}}$. As $F$ agrees with $f$ on an open subset of $\Omega, F$ is the holomorphic extension of $f$ to $D^{n+1}$.

Proof of Lemma 1.4. Consider the smooth family of analytic discs $\left\{\mathcal{A}_{t}\right\}_{t \in[-1,1]}$ given by

$$
\mathcal{A}_{t}(z)=(z, 2 t-z) \text {. }
$$

Notice that $\mathcal{A}_{t}(\bar{D}) \cap S=\left\{(t+i y, t-i y) \mid y \in\left[-\sqrt{1-t^{2}}, \sqrt{1-t^{2}}\right]\right\}$.

Consider the case when $t \geq 0$. If $t \leq x \leq 1$ and $x^{2}+y^{2}=1$, then $|2 t-(x+i y)| \leq 1$. Thus, the sets

$$
\mathcal{S}_{t}^{+}=\left\{\mathcal{A}_{t}(x+i y) \mid t \leq x \leq 1, x^{2}+y^{2} \leq 1\right\}, \quad t \in[0,1],
$$

are compact sets, with $\partial \mathcal{S}_{t}^{+} \subseteq \omega$, and $\mathcal{S}_{t}^{+} \subseteq \omega$ for $t$ close to 1 . By the Kontinuitätsatz, therefore, $\left.f\right|_{V}$ extends holomorphically to a neighbourhood of $\{(z=$ $\left.x+i y,-z) \in \mathbb{C}^{2}|0 \leq x \leq 1| z \mid, \leq 1\right\}\left(=\mathcal{S}_{0}^{+}\right)$.

Similarly, by considering the sets

$$
\mathcal{S}_{t}^{-}=\left\{\mathcal{A}_{t}(x+i y) \mid-1 \leq x \leq t, x^{2}+y^{2} \leq 1\right\}, \quad t \in[-1,0],
$$

we can show that $\left.f\right|_{V}$ extends holomorphically to a neighbourhood of the graph of the (holomorphic) function $\psi(z)=-z$. Call this function $\tilde{f}$. We are now in the 
situation of the classical Hartogs phenomenon; hence $\tilde{f}$ extends holomorphically to $D^{2}$.

Proposition 1.2 clearly follows from the above theorem. We state one more corollary.

Corollary 1.5. Let $S$ be the graph of $\left(\phi_{1}, \ldots, \phi_{n}\right): D \rightarrow \mathbb{C}^{n}$, a $D^{n}$-valued conjugate holomorphic map. With $\Omega$ as in Theorem 1.3, if $f \in \mathcal{O}(\Omega)$, then $f$ extends holomorphically to $D^{n+1}$.

\section{A generalization}

An examination of the proof of Theorem 1.3 reveals that the method of treating the conjugate variable $\bar{z}$ as an independent variable $\xi$ can be generalized to arbitrary $D$-valued continuous functions. This results in Theorem 2.1 below. Its proof is nearly similar to that of Theorem 1.3. At one stage, however, it appeals to the Hartogs-Chirka extension theorem - something that could be avoided in proving all of the preceding results.

Theorem 2.1. Let $u \in \mathcal{C}(\bar{D} ; D)$. Let $S$ be the graph of $\left(\phi_{1}, \ldots, \phi_{n}\right): \bar{D} \rightarrow \mathbb{C}^{n}$, each $\phi_{k}$ having the form:

$$
\phi_{k}(z)=\sum_{j=1}^{N} h_{j}^{(k)}(z) u(z)^{j},
$$

where $h_{j}^{(k)} \in \mathcal{O}(D) \cap \mathcal{C}(\bar{D}, \mathbb{C})$ with the property

$$
\sup _{z \in \bar{D}}\left[\sum_{j=1}^{N}\left|h_{j}^{(k)}(z)\right|\right]<1, \quad k=1, \ldots, n .
$$

With $\Omega$ as in Theorem 1.3, if $f \in \mathcal{O}(\Omega)$, then $f$ extends holomorphically to $D^{n+1}$.

Proof. Write $\Phi_{k}(z, \xi)=\sum_{j=1}^{N} h_{j}^{(k)}(z) \xi^{j}$. Exactly as in the proof of Theorem 1.3, there is an $\epsilon_{0}, 0<\epsilon_{0}<\delta / 2$, sufficiently small that for $\left|w_{k}\right|<\epsilon_{0}, k=1, \ldots, n$, $|z|<1$ and $|\xi-u(z)|<\epsilon_{0}$,

$$
\left|\left\{w_{k}+\Phi_{k}(z, \xi)\right\}-\Phi_{k}(z, u(z))\right|<\delta, \quad k=1, \ldots, n .
$$

So, for each fixed $w \in D_{\epsilon_{0}}^{n}$, for $|z|<1,|\xi-u(z)|<\epsilon_{0}$, we can define

$$
H(z, \xi, w)=f\left(z, w_{1}+\Phi_{1}(z, \xi), \ldots, w_{n}+\Phi_{n}(z, \xi)\right) .
$$

Furthermore, the bounds on $h_{j}^{(k)}$ and $u$ allow us to define

$$
(z, \xi) \mapsto H(z, \xi, w), \quad w \text { fixed, }\left|w_{k}\right|<\epsilon_{0},
$$

in $\left\{|z|<1,|\xi-u(z)|<\epsilon_{0}\right\} \cup\{1-\delta<|z|<1+\delta,|\xi| \leq 1\} \subseteq \mathbb{C}^{2}$ as a holomorphic function.

By the Hartogs-Chirka extension theorem (i.e. Theorem 1.1), we can define a function $\tilde{H}$ such that for each $w \in D_{\epsilon_{0}}^{n}$,

$$
(z, \xi) \mapsto \tilde{H}(z, \xi, w)
$$

holomorphically extends the function given by (ii) to $D^{2}$.

By the same argument as in Theorem 1.3, we can define the holomorphic function

$$
\tilde{f}(z, w)=\tilde{H}\left(z, 0, w_{1}, \ldots, w_{n}\right)
$$


where $\tilde{f}$ is defined in a neighbourhood of $\left(\partial D \times D^{n}\right) \cup\left\{w_{1}=\ldots=w_{n}=0\right\}$. By the classical theorem of Hartogs, $\tilde{f}$ extends to $F \in \mathcal{O}\left(D^{n+1}\right)$ and, by the same argument as in the proof of Theorem 1.3, $F$ agrees with $f$ on an open subset of $\Omega$. Thus, $F$ is the holomorphic extension of $f$ to $D^{n+1}$.

\section{ACKNOWLEDGEMENT}

The author wishes to thank Jean-Pierre Rosay for his helpful comments.

\section{REFERENCES}

1. E.M. Chirka, Generalized Hartogs' lemma and non-linear $\bar{\partial}$-equation, Complex analysis in contemporary mathematics (E.M. Chirka, ed.), Fasis, Moscow (in Russian) (to appear).

2. E.M. Chirka and J.-P. Rosay, Remarks on the proof of a generalized Hartogs lemma, Ann. Pol. Math. 70 (1998), 43-47. MR 2000a:32071

3. J.-P. Rosay, A counterexample related to Hartogs' phenomenon (A question by E. Chirka), Michigan Math. J. 45 (1998), 529-535. MR 2000a:32070

Department of Mathematics, University of Wisconsin-Madison, 480 Lincoln Drive, MADISON, Wisconsin 53706

E-mail address: bharali@math.wisc.edu 